Article

\title{
The CALIFA Survey: Exploring the Oxygen Abundance in the Local Universe
}

\author{
Sebastian F. Sánchez ${ }^{1, *}$, Laura Sánchez-Menguiano ${ }^{2,3}$, Raffaella Marino ${ }^{4}$, F. Fabian \\ Rosales-Ortega $^{5}$, Isabel Pérez ${ }^{3}$, Armando Gil de Paz ${ }^{4}$, Enrique Pérez ${ }^{2}$, C. Jakob Walcher ${ }^{6}$ \\ and Carlos López-Cobá ${ }^{1}$ \\ ${ }^{1}$ Instituto de Astronomía, Universidad Nacional Autonóma de México, A.P. 70-264, 04510 \\ México, México; E-Mail: carlos.lopezcoba@gmail.com \\ ${ }^{2}$ Instituto de Astrofísica de Andalucía (CSIC), Glorieta de la Astronomía s/n, Aptdo. 3004, \\ E-18080 Granada, Spain; E-Mails: lausan89@gmail.com (L.S.M.); eperez@iaa.es (E.P.) \\ ${ }^{3}$ Dpto. de Física Teórica y del Cosmos, Universidad de Granada, Facultad de Ciencias (Edificio \\ Mecenas), E-18071 Granada, Spain; E-Mail: isa@ugr.es \\ ${ }^{4}$ EI Campus Moncloa, UCM-UPM, Departamento de Astrofísica y CC. de la Atmósfera, Facultad de \\ CC. Físicas, Universidad Complutense de Madrid, Avda. Complutense s/n, 28040 Madrid, Spain; \\ E-Mails: ramarino@ucm.es (R.M.); agil@ucm.es (A.G.D.P.) \\ ${ }^{5}$ Instituto Nacional de Astrofísica, Óptica y Electrónica, Luis E. Erro 1, 72840 Tonantzintla, Puebla, \\ Mexico; E-Mails: f.rosales.ortega @ gmail.com \\ ${ }^{6}$ Leibniz-Institut für Astrophysik Potsdam (AIP), An der Sternwarte 16, D-14482 Potsdam, Germany; \\ jwalcher@aip.de
}

* Author to whom correspondence should be addressed; E-Mail: sfsanchez@astro.unam.mx; Tel.: +52-55-56-224-014.

Academic Editors: José Alfonso López Aguerri, Enrichetta Iodice and Alexei Moiseev Received: 23 September 2015 / Accepted: 22 October 2015 / Published: 5 November 2015

\footnotetext{
Abstract: We present here a review of the latest results on the spatially-resolved analysis of the stellar populations and ionized gas of disk-dominated galaxies based on Calar Alto Legacy Integral Field Area (CALIFA) data. CALIFA is an ongoing integral field spectroscopy (IFS) survey of galaxies in the Local Universe $(0.005<\mathrm{z}<0.03)$ that has already obtained spectroscopic information up to $\sim 2.5 r_{e}$ with a spatial resolution better than $\sim 1 \mathrm{kpc}$ for a total number of more than 600 galaxies of different morphological types, covering the color-magnitude diagram up to $\mathrm{M}_{\mathrm{R}}<-18$ mag. With nearly 2000 spectra obtained for each galaxy, CALIFA offers one of the best IFUdatasets to study the star
} 
formation histories and chemical enrichment of galaxies. In this article, we focus on the main results from the analysis of the oxygen abundances based on the study of ionized gas in $\mathrm{H}$ II regions and individual spaxels and their relation to the global properties of galaxies, using an updated/revised dataset with more galaxies and ionized regions. In summary, we have confirmed previous published results indicating that: (1) the $\mathcal{M}-\mathrm{Z}$ relation does not present a secondary relation to the star formation rate, when the abundance is measured at the effective radius; (2) the oxygen abundance presents a strong correlation with the stellar surface density ( $\Sigma$-Z relation); (3) the oxygen abundance profiles present three well-defined regimes: (i) an overall negative radial gradient between 0.5 and $2 r_{e}$, with a characteristic slope of $\alpha_{O / H} \sim-0.1 \mathrm{dex} / r_{e}$; (ii) a universal flattening beyond $>2 r_{e}$; and (iii) an inner drop at $<0.5 r_{e}$ that depends on mass; (4) the presence of bending in the surface brightness profile of disk galaxies is not clearly related to either the change in the shape of the oxygen abundance profile or the properties of the underlying stellar population. All of these results indicate that disk galaxies present an overall inside-out growth, with chemical enrichment and stellar mass growth tightly correlated and dominated by local processes and limited effects of radial mixing or global outflows. However, clear deviations are shown with respect to this simple scenario, which affect the abundance profiles in both the innermost and outermost regions of galaxies.

Keywords: ISM; galaxies; oxygen abundance

\section{Introduction}

We present here a brief summary of the status of the on-going Calar Alto Legacy Integral Field Area (CALIFA) survey with an emphasis on the results that have been recently published. In particular, we make a summary of the most relevant results found regarding the properties of $\mathrm{H}$ II regions discovered using this survey, focused on two main results: (i) the existence of a characteristic gradient of oxygen abundance irrespective of the properties of the galaxies; and (ii) the lack of a secondary correlation with the star formation in the mass-metallicity (oxygen abundance) relation.

\section{CALIFA: A Brief Introduction}

The Calar Alto Legacy Integral Field Area (CALIFA) survey [1] is an ongoing large project of the Centro Astronómico Hispano-Alemán at the Calar Alto observatory to obtain spatially-resolved spectra for 600 local $(0.005<\mathrm{z}<0.03)$ galaxies by means of integral field spectroscopy (IFS). CALIFA observations started in June 2010 with the Potsdam Multi-Aperture Spectrograph (PMAS), mounted to the 3.5-m telescope, utilizing the large $\left(74^{\prime \prime} \times 64^{\prime \prime}\right)$ hexagonal field-of-view (FoV) offered by the PPak fiber bundle [2,3]. PPak was created for the Disk Mass Survey [4]. Each galaxy is observed using two different setups, an intermediate spectral resolution one (V 1200, $R \sim 1650$ ) that covers the blue range of the optical wavelength range (3700-4700 $\AA$ ) and a low-resolution one (V 500, $R \sim 850)$ that covers the first order of the optical wavelength range (3750-7500 $\AA$ ). A diameter-selected sample of 
939 galaxies was drawn from the seventh data release of the Sloan Digital Sky Survey (SDSS), which is described in [5]. From this mother sample, the 600 target galaxies are randomly selected.

Combining the techniques of imaging and spectroscopy through optical IFS provides a more comprehensive view of individual galaxy properties than any traditional survey. CALIFA-like observations were collected during the feasibility studies [6,7] and the PPak IFS Nearby Galaxy Survey (PINGS, [8]), a predecessor of this survey. The first results based on those datasets already explored their information content (e.g., $[8,9]$ ).

Compared to other IFS surveys, CALIFA offers a unique combination of: (i) a sample covering a wide range of morphological types in a wide range of masses, sampling the color-magnitude diagram for $\mathrm{M}_{\mathrm{R}}>-18 \mathrm{mag}$; (ii) a large FoV, that guarantees covering the entire optical extension of the galaxies up to $2.5 r_{e}$ for $80 \%$ of the sample; and (iii) an accurate spatial sampling, with a typical spatial resolution of $\sim 1 \mathrm{kpc}$ for the entire sample, which allows one to spatially resolve the spectroscopic properties of most relevant structures in galaxies (spiral arms, bars, bulges, H II regions). The penalty for a better spatial sampling of the galaxies is the somehow limited number of galaxies in the survey (e.g., with respect to MaNGA and SAMI ). In terms of spectral resolution, only the blue wavelength range is sampled with a similar spectral resolution as these other two surveys.

As a legacy survey, one of the main goals of the CALIFA collaboration is to grant public access of the fully-reduced data cubes, in a sequence of data releases (DR). In November 2012, we delivered our first DR [10], comprising 200 data cubes corresponding to 100 objects (http://califa.caha.es/DR1/). After almost two years, and a major improvement in the data reduction, we presented our second data release [11], comprising 400 data cubes corresponding to 200 objects (http://califa.caha.es/DR2/), the first of October 2014. The third and final DR will be presented in spring 2016, and it will comprise more than 600 objects (1200 data cubes).

\section{CALIFA: Main Science Results}

The data products that can be derived from the IFU datasets obtained by the CALIFA survey comprise information on the stellar populations, ionized gas, mass distribution and stellar and gas kinematics. Similar data products are derived for any of the indicated projects: Atlas3D [12], MaNGA [13] or SAMI [14]. In summary, they comprise a panoramic view of the spatially-resolved spectroscopic properties of these galaxies.

Different science goals have been already addressed using this information: (i) New techniques have been developed to understand the spatially-resolved star formation histories (SFH) of galaxies [15,16]. We found solid evidence that mass assembly in typical galaxies happens from inside-out [17]. The SFH and chemical enrichment of bulges and early-type galaxies are fundamentally related to the total stellar mass, while for disk galaxies, it is more related to the local stellar mass density [18,19]; negative age gradients indicate that the quenching is progressing outwards in massive galaxies [18], and age and metallicity gradients suggest that galaxy bars have not significantly altered the SFH of spirals [20]; finally, we explore the spatially-resolved stellar populations across the Hubble sequence [19] and how mergers influence the assembly of blue elliptical galaxies [21]. (ii) We explore the origin of the low intensity, LINER -like, ionized gas in galaxies. These regions are clearly not related to star formation 
activity or to AGN activity [22]. They are most probably related to post-AGB ionization in many cases [23,24] and [25]. (iii) We explore the aperture and resolution effects on the data. CALIFA provides a unique tool to understand the aperture and resolution effects in larger single-fiber (like SDSS) and IFS surveys (like MaNGA, SAMI). We explored the effects of the dilution of the signal in different gas and stellar population properties [26] and proposed a new empirical aperture correction for the SDSS data [27]. (iv) CALIFA is the first IFU survey that allows gas and stellar kinematic studies for all morphologies with enough spectroscopic resolution to study: (1) the kinematics of the ionized gas [28]; (2) the effects of bars in the kinematics of galaxies [29,30]; (3) the effects of the interaction stage on the kinematic signatures [31]; (4) and measure the bar pattern speeds in late-type galaxies [32]. (v) We extend the measurements of the angular momentum of galaxies to previously-unexplored ranges of morphology and ellipticity [33], and propose a new dynamical classification scheme for galaxies [34]. (vi) We explore in detail the effects of galaxy interaction in the enhancement of the star formation rate and the ignition of galactic outflows [35,36]. (vii) We study the nature of the progenitors of SNe [37]. (viii) Finally, we explore the star formation indicators for extended objects and the capability of using $\mathrm{H} \alpha$ as a good SFR tracer [38]. Further results based on the analysis of the $\mathrm{H}$ II regions will be discussed in the next section.

\section{Results of Our Studies of the H II Regions}

The project to derive the properties of the H II regions in galaxies in the Local Universe using IFS data was initiated based on the data from the PINGS survey [39]. This survey acquired IFS mosaic data for a dozen medium-sized nearby galaxies. In $[8,40]$, we studied in detail the ionized gas and $\mathrm{H}$ II regions of the largest galaxy in the sample (NGC 628). The main results of these studies are included in the contribution by Rosales-Ortega in this volume. We then continued the acquisition of IFS data for a larger sample of visually-classified face-on spiral galaxies [6], as part of the feasibility studies for the CALIFA survey [1]. The spatially-resolved properties of a typical galaxy in this sample, UGC9837, were presented by [7].

In [41], we presented a new method to detect, segregate and extract the main spectroscopic properties of $\mathrm{H}$ II regions from IFS data (HIIEXPLORER). Figure 1, left-panel, illustrates how this method works, showing the $\mathrm{H} \alpha$ intensity map of NGC 6155 as observed by CALIFA, together with the segmentation map of the ionized regions detected. A preliminary catalog of $\sim 2600 \mathrm{H}$ II regions and aggregations extracted from 38 face-on $\left(i<45^{\circ}\right)$ spiral galaxies compiled from the PINGS and CALIFA feasibility studies was presented. We found a new local scaling relation between the stellar mass density and oxygen abundance, the so-called $\Sigma$-Z relation [9].

The same catalog allows us to explore the galactocentric radial gradient of the oxygen abundance [41]. Figure 1, right-panel, shows a typical radial abundance gradient for the galaxies within the CALIFA sample, derived using the $\mathrm{O} 3 \mathrm{~N} 2$ calibrator by $12+\log (\mathrm{O} / \mathrm{H})=8.73-0.32 \log (\mathrm{O} 3 \mathrm{~N} 2)$ [42]. We confirmed that up to $\sim 2$ disk effective radii $\left(r_{e}\right)$, there is a negative gradient of the oxygen abundance in all of the spiral galaxies analyzed. The gradient presents a very similar slope for all of the galaxies $\left(\sim-0.12 \pm 0.11 \mathrm{dex} / r_{e}\right)$, when the radial distances are measured in units of the disk effective radii. Beyond $\sim 2$ disk effective radii, our data show evidence of a flattening in the abundance for those galaxies 
that cover that galactocentric distance, as we will show later, consistent with several other spectroscopic explorations, based mostly on a few objects, e.g., [43].

In [44], we used the updated CALIFA catalog of $\sim 7000 \mathrm{H}$ II regions, extracted from 306 individual galaxies, to study the radial oxygen abundance gradient up to 3-4 disk effective radii. We confirmed that the abundance gradients present a common slope up to $\sim 2$ effective radii, with a distribution compatible with being produced by random fluctuations, for all galaxies when normalized to the disk effective radius of $\alpha_{O / H}=-0.1 \mathrm{dex} / r_{e}$ (using the $\mathrm{O} 3 \mathrm{~N} 2$ calibrator by [42]). No significant differences are found on the basis of the morphological type, presence or absence of bars, absolute magnitude and/or stellar mass. The only clear deviation from the common slope is seen in galaxies with evidence of interaction or undergoing a merging process. For these galaxies, the gradient is significantly flatter, in agreement with [45].
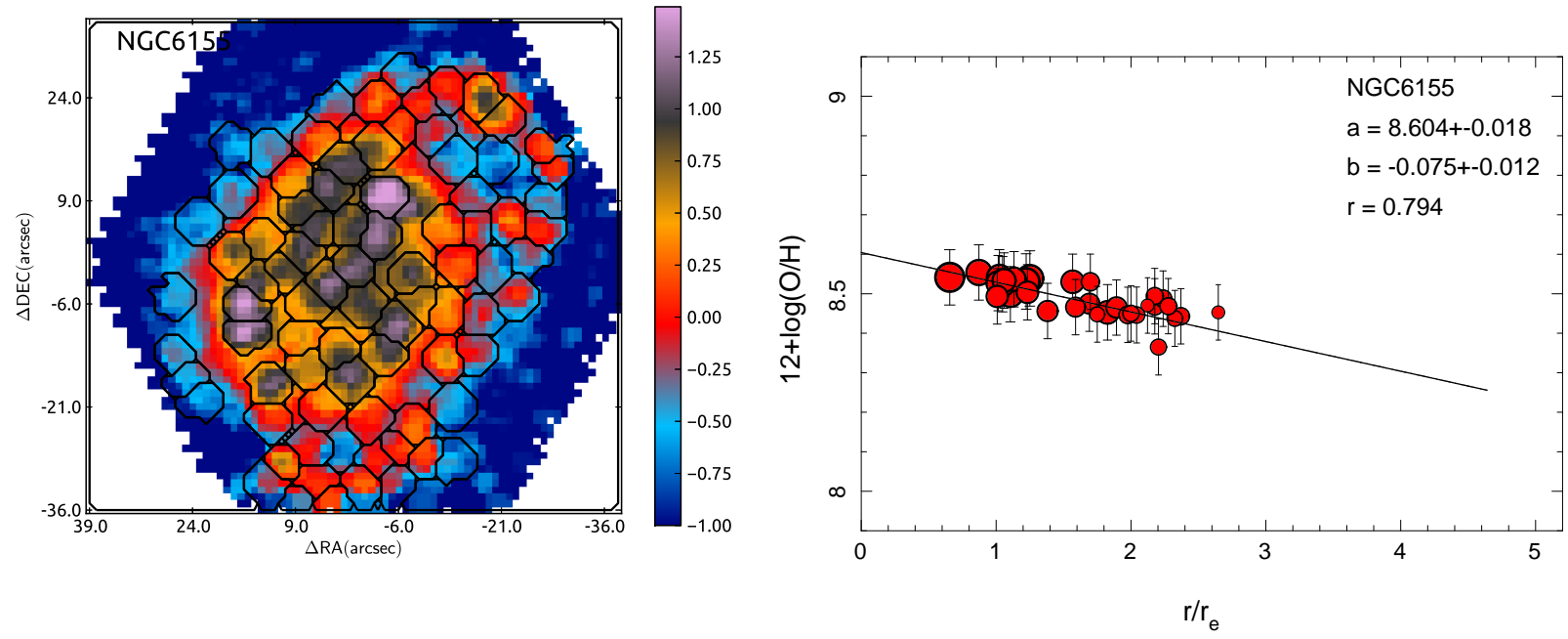

Figure 1. Left panel: integral field spectroscopy (IFS)-based $\mathrm{H} \alpha$ maps, in units of $10^{-16}$ erg s $\mathrm{s}^{-1} \mathrm{~cm}^{-2} \operatorname{arcsec}^{-2}$, derived for NGC 6155 (color image), together with the detected $\mathrm{H}$ II regions shown as black segmented contours; right panel: radial distribution of the oxygen abundance derived for the individual $\mathrm{H}$ II regions with abundance errors below 0.15 dex, as a function of the deprojected distance (i.e., corrected for inclination), normalized to the effective radius, for the same galaxy. The size of the circles is proportional to the $\mathrm{H} \alpha$ intensity.

To further explore these issues, we applied the same procedure to the IFS data provided by the CALIFA survey. In [46], we presented the first results based on the catalog of H II regions extracted from an enlarged sample of galaxies $(\sim 100)$. We studied the dependence of the $\mathcal{M}-\mathrm{Z}$ relation on the star formation rate. We found no secondary relation different from the one induced by the well-known relation between star formation and mass, contrary to what was claimed by other authors $[47,48]$ based on single-aperture spectroscopic data (SDSS). Although the reason for the discrepancy is still not clear, we postulated that simple aperture bias, like the one presented in previous datasets, may induce the reported secondary relation. Figure 2 presents an updated version of these results, including the last list of galaxies analyzed until January 2015 (271 galaxies from the CALIFA sample together with 31 galaxies from the CALIFA pilot studies). This sample of galaxies comprises all galaxies observed up to November 
2014 (492 objects), for which we have at least five H II regions/aggregations within a range between 0.5 and $2.1 r_{e}$ of galactocentric distances, thus those galaxies for which we have a well-defined oxygen abundance at the effective radius as defined in [46]. In total, the current sample is three-times larger than the one presented in our previous studies, with a much better coverage of the mass range.

The left panel of Figure 2 shows the $\mathcal{M}-Z$ relation found for these galaxies, with the color code indicating the integrated SFR for each galaxy. Notably, the stronger gradient in SFR is along the stellar mass, as expected for star-forming galaxies. Once subtracting the best fitted function to the $\mathcal{M}-\mathrm{Z}$ relation, the residuals of the abundance do not present any evident secondary relation to the SFR (right panel). Thus, the results presented in [46] are confirmed with a sample of galaxies enlarged by almost a factor of two. We tested the results using different calibrators for the oxygen abundance, including the O3N2 and N2 of both [42,49], the ONS by [50] and the counter-method by [51], without significant qualitative results.
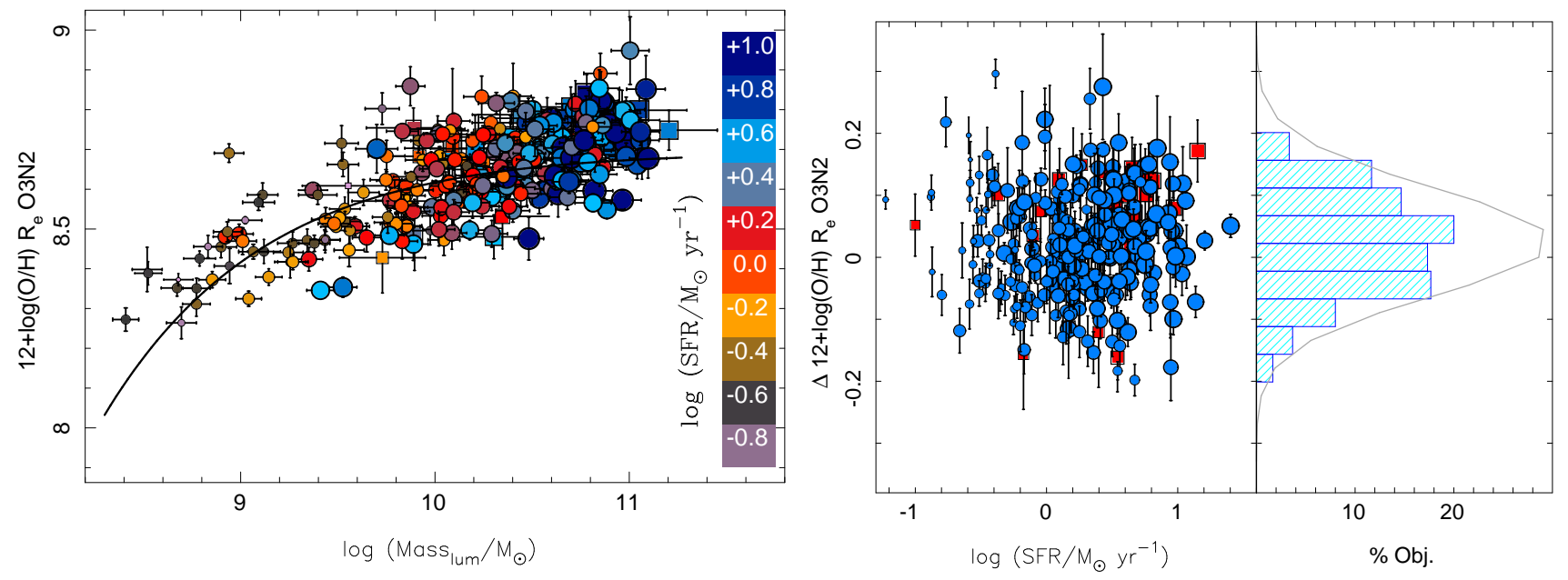

Figure 2. Left panel: distribution of the oxygen abundances at the effective radii as a function of the integrated stellar masses for the Calar Alto Legacy Integral Field Area (CALIFA) galaxies (271, circles), together with those from the CALIFA feasibility studies (31, squares). Right panel: distribution of the differential oxygen abundances with respect to the solid-line shown in the left panel (i.e., the dependence on the stellar mass), as a function of the integrated SFRfor the CALIFA galaxies.

We also confirmed the local $\Sigma-Z$ relation unveiled by [9], with a larger statistical sample of $\mathrm{H}$ II regions $(\sim 20,000)$. This nebular $\Sigma$-Z relation is flatter than the one derived for the average stellar populations [52], but both agree for the younger stars, as expected if the most recent stars are born from the chemically-enriched ISM. Figure 3 shows the most recently-derived $\Sigma$-Z relation using the $\sim 9000 \mathrm{H}$ II regions detected in the 492 CALIFA galaxies described before. The dispersion around the average relation is $\sim 0.08$ dex, just slightly larger than the one reported for the global $\mathcal{M}-\mathrm{Z}$ relation, in agreement with the results presented in $[8,46]$. In addition, we derived the same relation using a spaxel-by-spaxel selection of the star-forming regions, presented in [53], that comprise a total of nearly one million regions. Figure 3, right panel, shows the derived distribution using this dataset, which presents general good agreement with the $\Sigma$-Z relation derived using the $H$ II regions. The main difference is that the relation in the right panel seems to be slightly steeper than the relation in the left 
panel. This is induced by an aperture effect, since the figure on the left corresponds to a smoothing of the figure on the right for apertures of the typical size of the H II regions $\left(3^{\prime \prime}-4^{\prime \prime}\right)$.
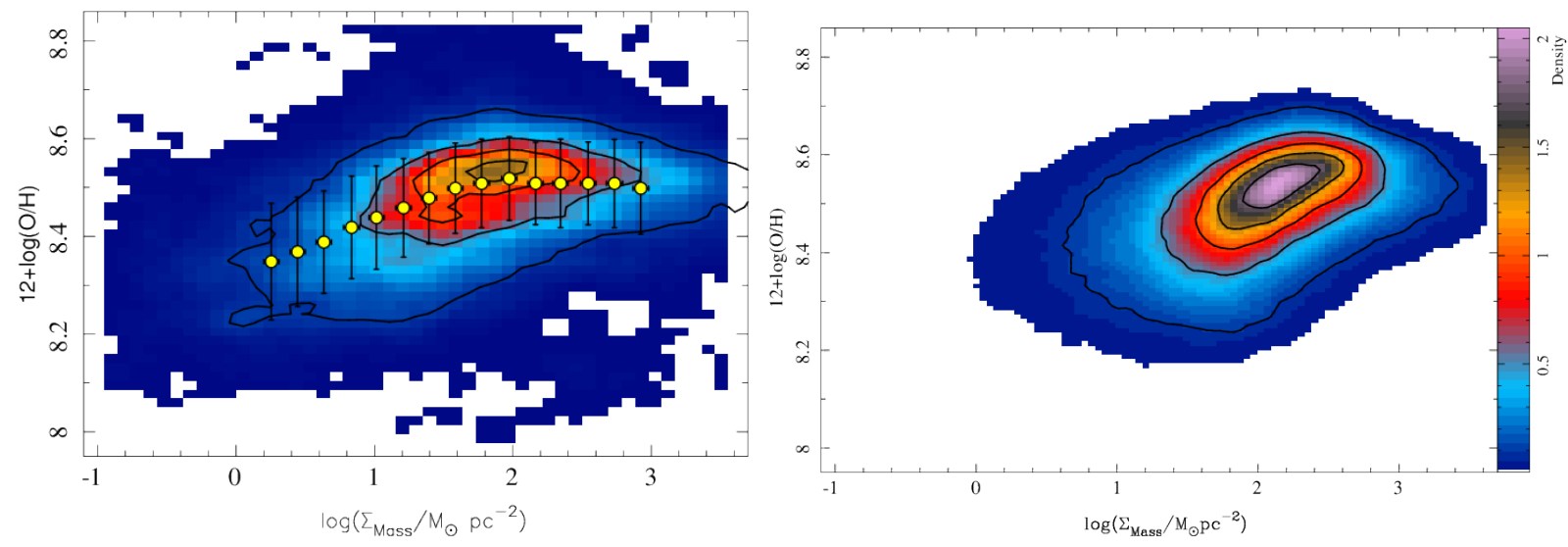

Figure 3. Left panel: distribution of the oxygen abundance along the stellar mass surface density for the $\sim 9000 \mathrm{H}$ II regions detected for the galaxies presented in Figure 2 . The image and contours show the density of points along the distribution, with the first contour encircling $95 \%$ of the regions and the percentage decreasing by $20 \%$ for each successive contour. The yellow solid points indicate the average abundance for a set of successive bins of 0.2 dex in stellar mass density, with the error bars indicating the standard deviation of the abundance distribution for each bin (with a typical value of $\sim 0.08 \mathrm{dex}$ ). Right panel: this shows the same distribution of the $\sim 1$ million spaxels extracted from the same CALIFA galaxies for which ionization conditions is dominated by star formation, following the criteria outlined in [46]. Image and contours present the same density distribution.

These results agree with the main conclusion of our previous study [41], where a limited sample of 38 face-on spiral galaxies was analyzed, using similar methods. In a companion article [20], we analyze the radial gradient of stellar metallicity, where we have found consistent results. New studies seem to confirm the existence of a characteristic or benchmark slope in the abundance gradient of disk galaxies [54].

Beyond $\sim 2 r_{e}$, the data confirms our previous result of the presence of a flattening in the abundance in most galaxies with detected $\mathrm{H}$ II regions at this radial distance. A change in the slope of the radial gradient of oxygen abundance has been reported by several authors, e.g., [8,43,55], although with less significant numbers. On the other hand, in the inner regions, the abundance gradient seems to present a drop or flattening for some galaxies, although this does not seem to be a general property of the abundance gradients.

All of these results were recently confirmed by a study performed both using the H II regions and the ionized gas spaxel-by-spaxel Sánchez-Menguiano et al. [53]. In that study a detailed analysis was performed of the overall shape of the abundance gradient, restricting our sample to the face-on spiral galaxies within the CALIFA survey (122 objects). The total sample of individual abundance values comprises 185,000 spaxels and $7100 \mathrm{H}$ II regions/aggregations, distributed across the optical extension of these galaxies. It was found that the abundance profiles present three well-defined ranges with different behaviors: (1) within $\sim 0.5-2 r_{e}$, the abundance profile presents a negative gradient with a 
common slope ranging between $-0.07 \mathrm{dex} / r_{e}$ and $-0.14 \mathrm{dex} / r_{e}$ depending on the calibrator adopted; (2) beyond $>2 r_{e}$, all galaxies seem to present a flattening in the abundance gradient, irrespective of their properties (morphological type, mass, luminosity); and (3) within $<0.5 r_{e}$, some galaxies present a drop/flattening in the abundance distribution, deviating from the overall negative gradient, which seems to depend on the stellar mass of the galaxy (being more intense at higher masses). We should note here that the mixing of ionization between star formation and diffuse emission does not significantly affect the result due to the selection criteria, which impose a minimum EW $(\mathrm{H} \alpha)$ of $6 \AA$ for the selected spaxels (and H II aggregations), well above the typical $\mathrm{EW}(\mathrm{H} \alpha)<3 \AA$ of the diffuse ionization. That selection criteria implies that for the same continuum level, the diffuse ionization contributes always less than $50 \%$ to the total flux and typically less than $10 \%$. We repeated the analysis restricting the selection to those spaxels with an $\mathrm{EW}(\mathrm{H} \alpha)>20 \AA$ without any significant variation in the results.

Figure 4 illustrates these results, showing the common abundance gradient (left panel), very similar to the one reported in [44], and the different shapes in the abundance profile at different masses (right panel), where the three regimes described before are evident. For this particular case, the O3N2 calibrator proposed by [49] was adopted $(12+\log (\mathrm{O} / \mathrm{H})=8.50-0.22 \log (\mathrm{O} 3 \mathrm{~N} 2))$. However, we tested the qualitative results using not only this calibrator, but the O3N2 presented by [42], the ONS by [50] and the PYQZ [56].

We would like to enter into the discussion of the accuracy of the different calibrators; however, in all of our studies, we prefer those calibrators anchored to oxygen abundances derived using the direct method. Those calibrators per se avoid the intrinsic problem in any photo-ionization model-based calibrator, that is the double dependence of each line ratio with both the abundance and the ionization strength (plus many other physical parameters, like the relative abundance of nitrogen to oxygen, the corresponding sulfur to oxygen ratio, dust attenuation, geometry of the nebula, shape of the spectrum of the ionization source, and so on). The abundance calibrators based on the direct method provide a direct relation between the line ratios and the abundances, at the expense of a larger nominal dispersion in those relations and once assumed that the sample of $\mathrm{H}$ II regions used for the calibration cover a similar range of ionization conditions as the nebulae to be measured. Thus, the main advantage of this method is that the possible degeneracies between the abundance and the ionization parameter are intrinsically included, since both parameters present a well-known correlation, e.g., [57], a fact that has been well known since the early studies of the ionization conditions in H II regions [58]. Only the abundance derivations based on photoionization models should either impose somehow that correlation, e.g., [59], to derive reliable results or restrict the ionization source to reproduce that correlation (e.g., PYQZ), a problem that is avoided using calibrators based on the direct method, like O3N2 or ONS.

The second criticism is that the calibrators using the direct method hardly derive oxygen abundances above solar, in contrast with the metallicity-derived for the stellar populations using different methods, e.g., [19,52], not only in the Local Universe, but at larger redshifts. In the naive perception that chemical enrichment follows an instant recycling and a closed-box model, gas and stellar metallicity should be tightly linked, and they can only increase with time; therefore, the oxygen abundances derived using the direct method should be biases, since they cannot reproduce the supra-solar metallicities observed in some stellar populations. This criticism, while following the common sense, is basically wrong. It is true that the first generations of stars enrich the ISM fast and that gas-phase metallicity grows quickly. 
However, most of the chemical enrichment models predict a decrease of the gas-phase metallicity as metal-poor gas (or even pristine gas) is accreted by the galaxy, in particular in the last $8 \mathrm{~Gy}$, when the galaxy evolution has been dominated by quiescent processes. Since stars are metal traps, they exhibit an abundance of gas when they were formed, and since most of the stellar continuum in the optical range is dominated by main-sequence stars, they are basically showing the average metallicity of the gas $\sim 4$ Gy ago, not the current one that could well be lower than that of the average stellar population.
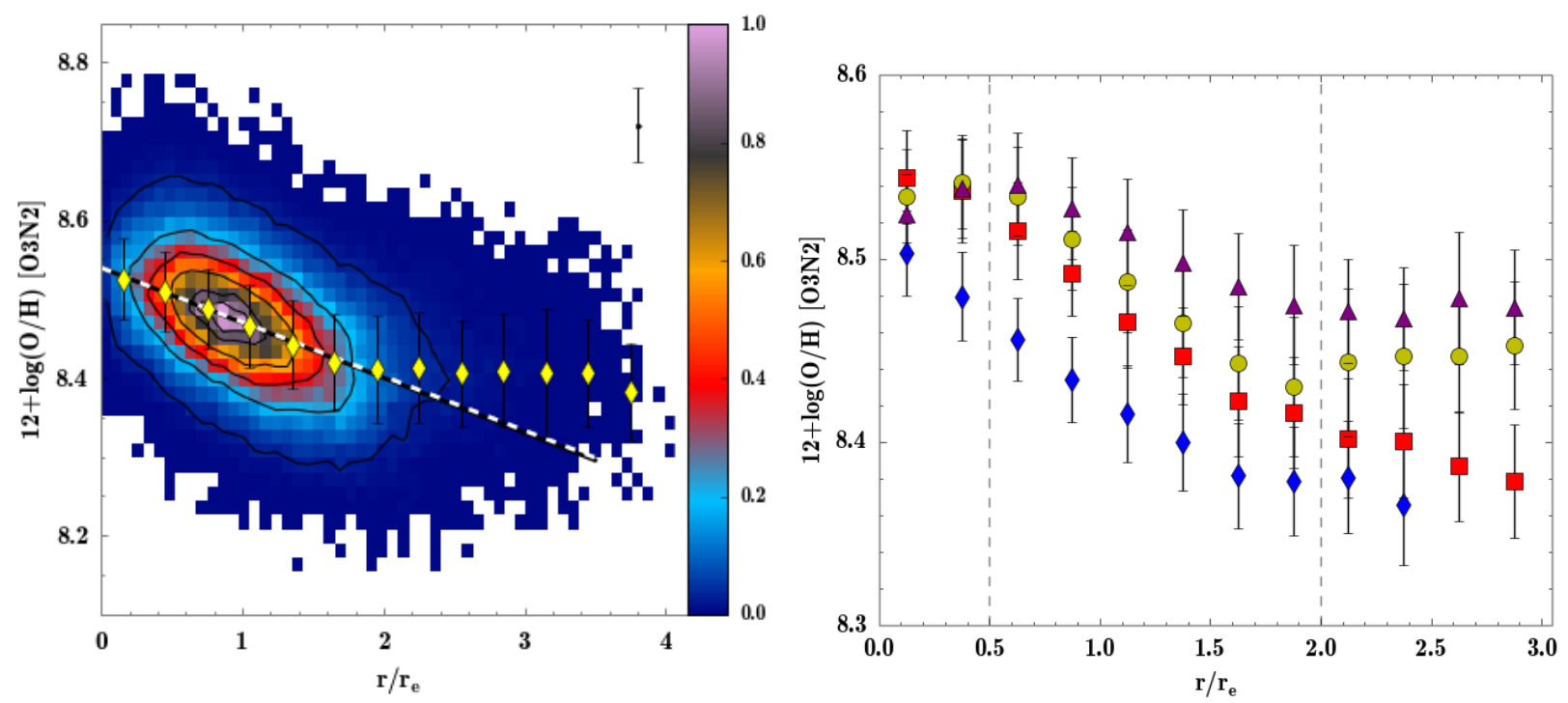

Figure 4. Left panel: radial density distribution of the oxygen abundance after re-scaling the oxygen abundances of each galaxy following the $\mathcal{M}-\mathrm{Z}$ relation derived in [46]: i.e., the abundance gradient for each galaxy was subtracted by the predicted value corresponding to its mass as derived using the $\mathcal{M}-\mathrm{Z}$ relation, and then, the average abundance for all the galaxies was added, to scale the gradient to an average value. The outermost contour encircles $95 \%$ of the total number of spaxels, decreasing $20 \%$ in each consecutive contour. The yellow diamonds represent the mean oxygen abundance values, with the error bars indicating the corresponding standard deviations, for bins of $0.25 r_{e}$. The solid-black line represents the error-weighted linear fit derived for those mean values within the range between 0.5 and $2.0 r_{e}$ and the dashed-white line represents the linear relation corresponding to the characteristic values of the zero-points and slopes for the complete sample. Right panel: mean oxygen abundance radial profiles derived considering galaxies in four different bins according to their integrated stellar mass. The limits of the bins were chosen to ensure a similar number of elements in each bin: $\log (\mathrm{M} / \mathrm{M} \odot)<10.1$, blue diamonds; $10.1<\log (\mathrm{M} / \mathrm{M} \odot)<10.35$, red squares; $10.35<\log (\mathrm{M} / \mathrm{M} \odot)<10.65$, yellow dots; $\log (\mathrm{M} / \mathrm{M} \odot)>10.65$, purple triangles. Dashed vertical lines delimit the three different regimes in the oxygen abundance profiles.

Finally, for most of our studies, we adopt the $\mathrm{O} 3 \mathrm{~N} 2$ calibrator instead of more refined ones, like the ONS and the counter methods described before, for the following reasons: (i) it uses two pairs of line ratios that are very near in wavelength, and therefore, it is basically independent of the dust attenuation; (ii) it does not use just one pair of line ratios, like N2, which could be more sensitive to the 
relative abundance between nitrogen and oxygen; (iii) it does not use [OII], which is an emission line at the edge of the spectral regime covered by CALIFA (and most of the spectroscopic surveys at low-z), which normally present the largest problems in the spectrophotometric calibration; in addition, it is a non-resolved doublet (to our resolution), and therefore, the derivation of its flux is in many cases less accurate than the other two; (iv) it does not use the [SII] doublet that suffers from the same problems of [OII], being at the edge of the covered spectral range and being a doublet (this time resolved), but in addition, it can be affected by the telluric absorptions (at $\sim 6860 \AA$ ) and the water vapor vibrational lines of the atmosphere. In summary, although the $\mathrm{O} 3 \mathrm{~N} 2$ calibrator many present a nominal dispersion of $\sim 0.08$ dex, being less accurate than the nominal one presented by other calibrators, since it uses a lower number of emission lines, stronger ones and those less affected by spectrophotometric inaccuracies and dust attenuation issues, the final accuracy of the derived abundance used to be much better than the one provided by more complex calibrators.

The shape of the profile of the abundance gradient is directly related to the chemical enrichment processes and the secular evolution in galaxies. In a recent study [60], we investigate the relation between the oxygen abundance gradients in the outer disk and the presence of breaks in the surface brightness profiles and broad-band colors, for a large sample comprising 350 CALIFA galaxies. Galaxies were classified on the basis of the presence of a bending in their surface brightness profile in the three usual types (Type I, with no bending, Type II with a down-bending and Type III with an up-bending). For Type II, the presence of a U-shape in the color was confirmed, in agreement with the location of the bending. However, this reddening seems to be correlated with the flattening in the abundance gradient only for the low mass galaxies. Mass seems to affect more the shape of the abundance profile than the presence of a bending (in agreement with the results shown in Figure 4). Only for Type III galaxies, there seems to be a correlation between the change in the color and the abundance gradient, which could be explained as a downsizing effect on the population of Type III galaxies that enhances the inside-out growth in these galaxies. On the other hand, recent results by [61], based on the analysis of the star formation history of spiral galaxies with different surface brightness profile types, extracted from the CALIFA sample, found that the U-shape in the age distribution is found irrespective of the profile type. This seems to be the first clear evidence of a decoupling of the mechanisms that generate the bending in the profile and the evolution of the stellar populations.

\section{Evidence for an Inside-Out Growth}

Along this article, we describe the different results we have obtained in our studies of H II regions using IFS. Our main results can be summarized as follows:

- The updated analysis presented here does not reveal any evidence of a secondary relation between the $\mathcal{M}-\mathrm{Z}$ relation with the star formation rate, different from the well-known relation between the SFR and the stellar mass for star-forming galaxies. This result is based on a sample three-times larger than the one analyzed by [46] and with a much wider and better coverage in masses.

- The gas abundance is strongly correlated with the stellar surface mass density ( $\Sigma$-Z relation), following a correlation similar to the $\mathcal{M}-\mathrm{Z}$ relation, that expands over three orders of magnitude in 
stellar surface density. Therefore, the stellar mass growth and the oxygen abundance enrichment are tightly related. Since the first grows inside-out, the second follows a similar trend.

- The gas abundance presents a radial profile with three well-defined regimes: (1) An overall negative radial gradient, with more metal-rich H II regions in the inner areas and more metal-poor ones in the outer ones between 0.5 and $2 r_{e}$. Independently of the galaxy properties, all galaxies present a common slope for this gradient (when the galactocentric distance is normalized to the effective radius of the disk) with a value of $\alpha_{O / H}=-0.07 \mathrm{dex} / r_{e}$ and $-0.14 \mathrm{dex} / r_{e}$ (depending on the oxygen abundance calibrator adopted). (2) Beyond $>2 r_{e}$, all galaxies seem to present a flattening in the abundance gradient irrespective of their properties (morphological type, mass, luminosity). (3) Within $<0.5 r_{e}$, some galaxies present a drop/flattening in the abundance distribution, deviating from the overall negative gradient, which seems to depend on the stellar mass of the galaxy (being more intense at higher masses).

- The presence of a bending in the surface brightness profile of disk galaxies is not clearly related to either the change in the shape of the oxygen abundance profile or the properties of the underlying stellar population. Only for Type III disk galaxies is it possible to describe a trend between some of these properties.

The existence of a universal radial decrease in the oxygen abundance has been already reported in many previous studies e.g., [8,41,43,55,62-64]. This observational property is compatible with our current understanding of the formation and evolution of spiral galaxies, e.g., [65] and the references therein. Gas accretion brings gas into the galactic center, where it first reaches the required density to ignite star formation. Thus, the inner regions are populated by older stars; they have undergone a faster gas reprocessing, and galaxies experience an inside-out mass growth [66,67]. Both the extinction-corrected color gradients in nearby galaxies [68] and the weak dependence of the mass-size relation with redshift [69-71] support an inside-out scenario for the evolution of disks. Recent results, based on the analysis of the star formation history of CALIFA data, found undisputed evidence of the inside-out growth of the stellar mass in galaxies [17], at least for galaxies more massive than $10^{10} \mathrm{M} \odot$. These results are also supported by the radial distribution of the stellar ages found for the same dataset [18].

However, the described characteristic slope for the abundance gradient, independent of many of the properties of the galaxies, was only recently proposed [41]. This result imposes a more severe restriction on our current understanding of how disk galaxies grow. In essence, it agrees with the recently-proposed $\Sigma$-Z relation [9] that links the gas abundance with the mass density of the underlying stellar population. It describes how the stellar mass and the gas abundance, both fundamental products of the star formation history, grow consistently in disk galaxies, from the center to the outer parts. Together with the $\mathcal{M}-\mathrm{Z}$ relation, they indicate that more massive galaxies (that trace the strongest potential well) form before and faster, accumulating more stellar mass and more metals. The presence of a common gradient in the abundance indicates that all disk-dominated galaxies of the same disk effective radius (hence, same disk mass) build up their metal content at a given normalized radius with similar efficiency.

The common slope suggests that the chemical evolution of galaxies is very similar in all disk galaxies, being compatible with a closed-box model. The classical closed-box model considers that each radial bin of a galaxy comprises primordial gas from which stars are born, live all their life-time and die 
in situ, according to some SFR and IMF prescriptions [72]. Therefore, if the amount of primordial gas is proportional to the depth of the potential well and the efficiency of the SFR is the same for all galaxies, both the stellar mass and the enrichment would be just proportional to the time, for a given halo mass. The lack of dependence of the $\mathcal{M}-\mathrm{Z}$ and $\Sigma$-Z relations on the SFR (or the SFR surface density) also supports this scenario. Under this assumption, all galaxies should have a universal gradient of their oxygen abundance with its zero-point being proportional to the total mass.

However, it is well known that the closed-box model cannot predict the right fraction of metal-poor stars with respect to the observed metallicity distribution of nearby long-lived stars in the Milky Way, e.g., [73]. A more realistic model overcomes this problem by allowing the disk of galaxies to form via continuous accretion gas, driven by the gravitational force. This accretion can be compensated or even overcome for certain galaxies and certain periods by supernova explosions, e.g., [74]. However, the outflow of gas is not expected to feature in the history of most spiral galaxies and is usually neglected in the models [73]. This modified model is consistent with the described common radial gradient if the local gas recycling is faster than other timescales involved [75] and if the radial inflow is similar for those radial bins with the same stellar mass.

Despite this evidence, other authors consider that galactic winds play a fundamental role in the shaping of spiral galaxies, e.g., [76], and may be an essential ingredient in their formation. Galactic winds are normally associated with strong star formation events, but there is also evidence for such winds at more moderate SFR. They seem to be a ubiquitous event in high redshift galaxies, e.g., [77,78], and some theoretical work emphasizes that they are a fundamental ingredient in the evolution of galaxies, e.g., $[79,80]$. In particular, they may play a role in the enrichment of the intergalactic medium, although in most cases, they do not seem to escape the dark matter halo around the galaxy. However, our results indicate that gas flows seem to have a less relevant effect than anticipated in the shape of the chemical gradient in galaxies, i.e., their net effect is not evident.

Our results do not rule out the existence of radial gas motions and metal mixing; they, however, add new constraints on their net effect on the chemical abundance patterns of disk galaxies. Contrary to isolated disk galaxies, interacting/merging galaxies show a clearly flatter metallicity distribution, in agreement with $[81,82]$. This indicates that galaxy interactions can result in an effective mixing of metals. The absence of a clear difference in the radial abundance slopes of barred and unbarred galaxies suggests that bars: (i) do not significantly enhance the efficiency of metal mixing in galaxies; or (ii) produce a change roughly proportional in the gas abundance and stellar mass distribution, which compensate each other when normalized to the disk effective radius; or (iii) are of a temporary nature, and their lifetime is shorter than the timescale for chemical abundance mixing in galaxies.

In general, the properties of the ionized gas in late-type galaxies are consistent with a quiescent evolution, where gas recycling is faster than other time scales involved [75]. This would imply that galaxies seem to behave locally in a similar manner rather than globally, dominated by a radial mass distribution following the potential well of the matter, with an inside-out growth that is regulated by gas inflow and local downsizing star formation. Therefore, the dominant parameter that defines the amount of metals is the stellar mass, since both parameters are the consequence of an (almost) closed-box star formation process. 
The deviations from the simple monotonic decrease in the radial profile of the abundance gradient and the tight correlation between the stellar mass density and the oxygen abundance may indicate that the pure inside-out growth and pseudo-closed-box model are indeed valid only at first order. Processes that transfer gas across the galaxy, that allow the inflow of more metal-poor gas or gas recycling within the halo and quenching and/or a slow decrease of the star formation activity in the inner regions of the more massive galaxies should be fundamental ingredients in any realistic scenario that tries to describe the observed properties summarized in the current report.

\section{Acknowledgments}

Sebastian F. Sanchez thanks the support of the Mexican CONACyT Grant 180125, and DGAPA-PAPIIT Grant IA100815.

Authors thank to the rest of the CALIFA collaboration members for making possible this study.

\section{Author Contributions}

Sebastian F. Sánchez is the Principal Investigator of the CALIFA collaboration and has participated in all the studies summarized here. C. Jakob Walcher is the Project Scientist of the CALIFA collaboration and has participated in all the studies summarised here. In particular he has leaded the sample characterization. Laura Sanchez-Menguiano and R. Marino has worked on the production of the emission line catalog and generation of the oxygen abundances, together with F. Fabian Rosales-Ortega. Isabel Perez, Armando Gil de Paz, Enrique Perez and C. Lopez-Coba have participated in most of the studies summarized here, and have participated in both the technical analysis and the scientific discussion.

\section{Conflicts of Interest}

The authors declare no conflict of interest.

\section{References}

1. Sánchez, S.F.; Kennicutt, R.C.; Gil de Paz, A.; van de Ven, G.; Vílchez, J.M.; Wisotzki, L.; Walcher, C.J.; Mast, D.; Aguerri, J.A.L.; Albiol-Perez, S.; et al. CALIFA, the Calar Alto Legacy Integral Field Area survey. I. Survey presentation. Astron. Astrophys. 2012 , doi:10.1051/0004-6361/201117353.

2. Verheijen, M.A.W.; Bershady, M.A.; Andersen, D.R.; Swaters, R.A.; Westfall, K.; Kelz, A.; Roth, M.M. The Disk Mass project: science case for a new PMAS IFU module. Astron. Nachr. 2004, 325, 151-154.

3. Kelz, A.; Verheijen, M.A.W.; Roth, M.M.; Bauer, S.M.; Becker, T.; Paschke, J.; Popow, E.; Sánchez, S.F.; Laux, U. PMAS: The Potsdam Multi-Aperture Spectrophotometer. II. The Wide Integral Field Unit PPak. Publ. Astron. Soc. Pac. 2006, 118, 129-145.

4. Bershady, M.A.; Verheijen, M.A.W.; Swaters, R.A.; Andersen, D.R.; Westfall, K.B.; Martinsson, T. The DiskMass Survey. I. Overview. Astrophys. J. 2010, 716, 198-233. 
5. Walcher, C.J.; Wisotzki, L.; Bekeraitlęe, S.; Husemann, B.; Iglesias-Páramo, J.; Backsmann, N.; Ballesteros, J.B.; Catalán-Torrecilla, C.; Cortijo, C.; del Olmo, A.; et al. CALIFA: A diameter-selected sample for an integral field spectroscopy galaxy survey. 2014, arXiv:1407.2939.

6. Mármol-Queraltó, E.; Sánchez, S.F.; Marino, R.A.; Mast, D.; Viironen, K.; Gil de Paz, A.; Iglesias-Páramo, J.; Rosales-Ortega, F.F.; Vilchez, J.M. Integral field spectroscopy of a sample of nearby galaxies. I. Sample, observations, and data reduction. Astron. Astrophys. 2011, doi:10.1051/0004-6361/201117032.

7. Viironen, K.; Sánchez, S.F.; Marmol-Queraltó, E.; Iglesias-Páramo, J.; Mast, D.; Marino, R.A.; Cristóbal-Hornillos, D.; Gil de Paz, A.; van de Ven, G.; Vilchez, J.; Wisotzki, L. Spatially resolved properties of the grand-design spiral galaxy UGC 9837: A case for high-redshift 2-D observations. Astron. Astrophys. 2012, doi:10.1051/0004-6361/201016347.

8. Rosales-Ortega, F.F.; Díaz, A.I.; Kennicutt, R.C.; Sánchez, S.F. PPAK wide-field Integral Field Spectroscopy of NGC 628-II. Emission line abundance analysis. Mon. Not. R. Astron. Soc. 2011, 415, 2439-2474.

9. Rosales-Ortega, F.F.; Sánchez, S.F.; Iglesias-Páramo, J.; Díaz, A.I.; Vílchez, J.M.; Bland-Hawthorn, J.; Husemann, B.; Mast, D. A New Scaling Relation for H II Regions in Spiral Galaxies: Unveiling the True Nature of the Mass-Metallicity Relation. Astrophys. J. Lett. 2012, doi:10.1088/2041-8205/756/2/L31.

10. Husemann, B.; Jahnke, K.; Sánchez, S.F.; Barrado, D.; Bekeraitè, S.; Bomans, D.J.; Castillo-Morales, A.; Catalán-Torrecilla, C.; Cid Fernandes, R.; Falcón-Barroso, J.; et al. CALIFA, the Calar Alto Legacy Integral Field Area survey. II. First public data release. Astron. Astrophys. 2013, doi:10.1051/0004-6361/201220582.

11. García-Benito, R.; Zibetti, S.; Sánchez, S.F.; Husemann, B.; de Amorim, A.L.; Castillo-Morales, A.; Cid Fernandes, R.; Ellis, S.C.; Falcón-Barroso, J.; Galbany, L.; et al. CALIFA, the Calar Alto Legacy Integral Field Area survey. III. Second public data release. Astron. Astrophys. 2015, doi:10.1051/0004-6361/201425080.

12. Cappellari, M.; Emsellem, E.; Krajnovic, D.; McDermid, R.M.; Scott, N.; Verdoes Kleijn, G.A.; Young, L.M.; Alatalo, K.; Bacon, R.; Blitz, L.; et al. The Atlas3D project-I. A volume-limited sample of 260 nearby early-type galaxies: Science goals and selection criteria. 2010, arXiv:astro-ph.CO/1012.1551.

13. Bundy, K.; Bershady, M.A.; Law, D.R.; Yan, R.; Drory, N.; MacDonald, N.; Wake, D.A.; Cherinka, B.; Sánchez-Gallego, J.R.; Weijmans, A.M.; et al. Overview of the SDSS-IV MaNGA Survey: Mapping nearby Galaxies at Apache Point Observatory. Astrophys. J. 2015, doi:10.1088/0004-637X/798/1/7.

14. Croom, S.M.; Lawrence, J.S.; Bland-Hawthorn, J.; Bryant, J.J.; Fogarty, L.; Richards, S.; Goodwin, M.; Farrell, T.; Miziarski, S.; Heald, R.; et al. The Sydney-AAO Multi-object Integral field spectrograph. Mon. Not. R. Astron. Soc. 2012, 421, 872-893.

15. Cid Fernandes, R.; Perez, E.; Garcia Benito, R.; González Delgado, R.M.; de Amorim, A.L.; Sanchez, S.F.; Husemann, B.; Falcon Barroso, J.; Sanchez-Blazquez, P.; Walcher, C.J.; et al. Resolving galaxies in time and space: I: Applying STARLIGHT to CALIFA data cubes. 2013, arXiv:astro-ph.CO/1304.5788. 
16. Cid Fernandes, R.; González Delgado, R.M.; García Benito, R.; Pérez, E.; de Amorim, A.L.; Sánchez, S.F.; Husemann, B.; Falcón Barroso, J.; López-Fernández, R.; Sánchez-Blázquez, P.; et al. Resolving galaxies in time and space. II. Uncertainties in the spectral synthesis of data cubes. Astron. Astrophys. 2014, doi:10.1051/0004-6361/201321692.

17. Pérez, E.; Cid Fernandes, R.; González Delgado, R.M.; García-Benito, R.; Sánchez, S.F.; Husemann, B.; Mast, D.; Rodón, J.R.; Kupko, D.; Backsmann, N.; et al. The Evolution of Galaxies Resolved in Space and Time: A View of Inside-out Growth from the CALIFA Survey. Astrophys. J. Lett. 2013, doi:10.1088/2041-8205/763/1/L1.

18. González Delgado, R.M.; Pérez, E.; Cid Fernandes, R.; García-Benito, R.; de Amorim, A.L.; Sánchez, S.F.; Husemann, B.; Cortijo-Ferrero, C.; López Fernández, R.; Sánchez-Blázquez, P.; et al. The star formation history of CALIFA galaxies: Radial structures. Astron. Astrophys. 2014, doi:10.1051/0004-6361/201322011.

19. González Delgado, R.M.; García-Benito, R.; Pérez, E.; Cid Fernandes, R.; de Amorim, A.L.; Cortijo-Ferrero, C.; Lacerda, E.A.D.; López Fernández, R.; Vale-Asari, N.; Sánchez, S.F.; et al. The CALIFA survey across the Hubble sequence. Spatially resolved stellar population properties in galaxies. Astron. Astrophys. 2015, doi:10.1051/0004-6361/201525938.

20. Sánchez-Blázquez, P.; Rosales-Ortega, F.; Mendez-Abreu, J.; Pérez, I.; Sánchez, S.F.; Zibetti, S.; Aguerri, J.A.L.; Bland-Hawthorn, J.; Catalán-Torrecilla, C.; Cid Fernandes, R.; et al. Stellar Population gradients in galaxy discs from the CALIFA survey. 2014, arXiv:1407.0002.

21. Haines, T.; McIntosh, D.H.; Sánchez, S.F.; Tremonti, C.; Rudnick, G. Testing the modern merger hypothesis via the assembly of massive blue elliptical galaxies in the local Universe. Mon. Not. R. Astron. Soc. 2015, 451, 433-454.

22. Singh, R.; van de Ven, G.; Jahnke, K.; Lyubenova, M.; Falcón-Barroso, J.; Alves, J.; Cid Fernandes, R.; Galbany, L.; García-Benito, R.; Husemann, B.; et al. The nature of LINER galaxies: Ubiquitous hot old stars and rare accreting black holes. Astron. Astrophys. 2013, doi:10.1051/0004-6361/201322062.

23. Kehrig, C.; Monreal-Ibero, A.; Papaderos, P.; Vílchez, J.M.; Gomes, J.M.; Masegosa, J.; Sánchez, S.F.; Lehnert, M.D.; Cid Fernandes, R.; Bland-Hawthorn, J.; et al. The ionized gas in the CALIFA early-type galaxies. I. Mapping two representative cases: NGC 6762 and NGC 5966. Astron. Astrophys. 2012, doi:10.1051/0004-6361/201118357.

24. Papaderos, P.; Gomes, J.M.; Vilchez, J.M.; Kehrig, C.; Lehnert, M.D.; Ziegler, B.; Sánchez, S.F.; Husemann, B.; Monreal-Ibero, A.; García-Benito, R.; et al. Nebular emission and the Lyman continuum photon escape fraction in CALIFA early-type galaxies. 2013, arXiv:astro-ph.CO/1306.2338.

25. Gomes, J.M.; Papaderos, P.; Vilchez, J.M.; Kehrig, C.; Iglesias-Páramo, J.; Breda, I.; Lehnert, M.; et al. Spiral-like star-forming patterns in early-type galaxies Astron. Astrophys. 2015, accepted.

26. Mast, D.; Rosales-Ortega, F.F.; Sánchez, S.F.; Vílchez, J.M.; Iglesias-Paramo, J.; Walcher, C.J.; Husemann, B.; Márquez, I.; Marino, R.A.; Kennicutt, R.C.; et al. The effects of spatial resolution on integral field spectrograph surveys at different redshifts-The CALIFA perspective. Astron. Astrophys. 2014, doi:10.1051/0004-6361/201321789. 
27. Iglesias-Páramo, J.; Vílchez, J.M.; Galbany, L.; Sánchez, S.F.; Rosales-Ortega, F.F.; Mast, D.; García-Benito, R.; Husemann, B.; Aguerri, J.A.L.; Alves, J.; et al. Aperture corrections for disk galaxy properties derived from the CALIFA survey. Balmer emission lines in spiral galaxies. Astron. Astrophys. 2013, doi:10.1051/0004-6361/201321460.

28. Garcia-Lorenzo, B.; Marquez, I.; Barrera-Ballesteros, J.K.; Masegosa, J.; Husemann, B.; Falcón-Barroso, J.; Lyubenova, M.; Sánchez, S.F.; Walcher, J.; Mast, D.; et al. Ionized gas kinematics of galaxies in the CALIFA survey I: Velocity fields, kinematic parameters of the dominant component, and presence of kinematically distinct gaseous systems. $\mathbf{2 0 1 4}$, arXiv:1408.5765.

29. Barrera-Ballesteros, J.K.; Falcón-Barroso, J.; García-Lorenzo, B.; van de Ven, G.; Aguerri, J.A.L.; Mendez-Abreu, J.; Spekkens, K.; Lyubenova, M.; Sánchez, S.F.; Husemann, B.; et al. Kinematic alignment of non-interacting CALIFA galaxies. Quantifying the impact of bars on stellar and ionised gas velocity field orientations. Astron. Astrophys. 2014, 568, 1-30.

30. Holmes, L.; Spekkens, K.; Sánchez, S.F.; Walcher, C.J.; García-Benito, R.; Mast, D.; Cortijo-Ferrero, C.; Kalinova, V.; Marino, R.A.; Mendez-Abreu, J.; et al. The incidence of bar-like kinematic flows in CALIFA galaxies. Mon. Not. R. Astron. Soc. 2015, 451, 4397-4411.

31. Barrera-Ballesteros, J.K.; García-Lorenzo, B.; Falcón-Barroso, J.; van de Ven, G.; Lyubenova, M.; Wild, V.; Méndez-Abreu, J.; Sánchez, S.F.; Marquez, I.; Masegosa, J.; et al. Tracing kinematic (mis)alignments in CALIFA merging galaxies: Stellar and ionized gas kinematic orientations at every merger stage. 2015, arXiv:1506.03819.

32. Aguerri, J.A.L.; Méndez-Abreu, J.; Falcón-Barroso, J.; Amorin, A.; Barrera-Ballesteros, J.; Cid Fernandes, R.; García-Benito, R.; García-Lorenzo, B.; González Delgado, R.M.; Husemann, B.; et al. Bar pattern speeds in CALIFA galaxies. I. Fast bars across the Hubble sequence. Astron. Astrophys. 2015, doi:10.1051/0004-6361/201423383.

33. Falcón-Barroso, J.; Lyubenova, M.; van de Ven, G. Angular Momentum across the Hubble sequence from the CALIFA survey. In Proceedings of the IAU Symposium, Oxford, UK, 21-25 July 2014; Cappellari, M., Courteau, S., Eds.; International Astronomical Union: Paris, France, 2015; Volume 311, pp. 78-81.

34. Kalinova, V.; Colombo, D.; Rosolowsky, E.; van de Ven, G.; Lyubenova, M.; Falcón-Barroso, J.; Kannan, R.; Läsker, R.; Galbany, L.; García-Benito, R.; et al. Toward the Dynamical Classification of Galaxies: Principal Component Analysis of SAURON and CALIFA circular velocity curves. 2015, arXiv:1509.03352.

35. Wild, V.; Rosales-Ortega, F.; Falcón-Barroso, J.; García-Benito, R.; Gallazzi, A.; González Delgado, R.M.; Bekeraité, S.; Pasquali, A.; Johansson, P.H.; García Lorenzo, B.; et al. The Mice at play in the CALIFA survey. A case study of a gas-rich major merger between first passage and coalescence. Astron. Astrophys. 2014, doi:10.1051/0004-6361/201321624.

36. Barrera-Ballesteros, J.K.; Sánchez, S.F.; García-Lorenzo, B.; Falcón-Barroso, J.; Mast, D.; García-Benito, R.; Husemann, B.; van de Ven, G.; Iglesias-Páramo, J.; Rosales-Ortega, F.F.; et al. Central star formation and metallicity in CALIFA interacting galaxies. Astron. Astrophys. 2015, doi:10.1051/0004-6361/201425397. 
37. Galbany, L.; Stanishev, V.; Mourão, A.M.; Rodrigues, M.; Flores, H.; García-Benito, R.; Mast, D.; Mendoza, M.A.; Sánchez, S.F.; Badenes, C.; et al. Nearby supernova host galaxies from the CALIFA Survey: I. Sample, data analysis, and correlation to star-forming regions. 2014, arXiv:1409.1623.

38. Catalán-Torrecilla, C.; Gil de Paz, A.; Castillo-Morales, A.; Iglesias-Páramo, J.; Sánchez, S.F.; Kennicutt, R.C.; Pérez-González, P.G.; Marino, R.A.; Walcher, C.J.; Husemann, B.; et al. Star Formation in the Local Universe from the CALIFA sample. I. Calibrating the SFR using IFS data. 2015, arXiv:1507.03801.

39. Rosales-Ortega, F.F.; Kennicutt, R.C.; Sánchez, S.F.; Díaz, A.I.; Pasquali, A.; Johnson, B.D.; Hao, C.N. PINGS: The PPAK IFS Nearby Galaxies Survey. Mon. Not. R. Astron. Soc. 2010, 405, 735-758,

40. Sánchez, S.F.; Rosales-Ortega, F.F.; Kennicutt, R.C.; Johnson, B.D.; Diaz, A.I.; Pasquali, A.; Hao, C.N. PPAK Wide-field Integral Field Spectroscopy of NGC 628-I. The largest spectroscopic mosaic on a single galaxy. Mon. Not. R. Astron. Soc. 2011, 410, 313-340.

41. Sánchez, S.F.; Rosales-Ortega, F.F.; Marino, R.A.; Iglesias-Páramo, J.; Víłlchez, J.M.; Kennicutt, R.C.; Díaz, A.I.; Mast, D.; Monreal-Ibero, A.; García-Benito, R.; et al. Integral field spectroscopy of a sample of nearby galaxies. II. Properties of the $\mathrm{H}$ ii regions. Astron. Astrophys. 2012, doi:10.1051/0004-6361/201219578.

42. Pettini, M.; Pagel, B.E.J. [OIII]/[NII] as an abundance indicator at high redshift. Mon. Not. R. Astron. Soc. 2004, 348, L59-L63.

43. Bresolin, F.; Ryan-Weber, E.; Kennicutt, R.C.; Goddard, Q. The Flat Oxygen Abundance Gradient in the Extended Disk of M83. Astrophys. J. 2009, 695, 580-595.

44. Sánchez, S.F.; Rosales-Ortega, F.F.; Iglesias-Páramo, J.; Mollá, M.; Barrera-Ballesteros, J.; Marino, R.A.; Párez, E.; Sánchez-Blazquez, P.; González Delgado, R.; Cid Fernandes, R.; et al. A characteristic oxygen abundance gradient in galaxy disks unveiled with CALIFA. Astron. Astrophys. 2014, doi:10.1051/0004-6361/201322343.

45. Rupke, D.S.N.; Kewley, L.J.; Chien, L.H. Gas-phase Oxygen Gradients in Strongly Interacting Galaxies. I. Early-stage Interactions. Astrophys. J. 2010, 723, 1255-1271.

46. Sánchez, S.F.; Rosales-Ortega, F.F.; Jungwiert, B.; Iglesias-Páramo, J.; Vílchez, J.M.; Marino, R.A.; Walcher, C.J; Husemann, B.; Mast, D.; Monreal-Ibero, A.; et al. Mass-metallicity relation explored with CALIFA. I. Is there a dependence on the star formation rate? Astron. Astrophys. 2013, doi:10.1051/0004-6361/201220669.

47. Lara-López, M.A.; Cepa, J.; Bongiovanni, A.; Pérez García, A.M.; Ederoclite, A.; Castañeda, H.; Fernández Lorenzo, M.; Povic, M.; Sánchez-Portal, M. A fundamental plane for field star-forming galaxies. Astron. Astrophys. 2010, doi:10.1051/0004-6361/201014803.

48. Mannucci, F.; Cresci, G.; Maiolino, R.; Marconi, A.; Gnerucci, A. A fundamental relation between mass, star formation rate and metallicity in local and high-redshift galaxies. Mon. Not. R. Astron. Soc. 2010, 408, 2115-2127. 
49. Marino, R.A.; Rosales-Ortega, F.F.; Sánchez, S.F.; Gil de Paz, A.; Vílchez, J.; Miralles-Caballero, D.; Kehrig, C.; Pérez-Montero, E.; Stanishev, V.; Iglesias-Páramo, J.; et al. The O3N2 and N2 abundance indicators revisited: Improved calibrations based on CALIFA and $\mathrm{T}_{e}$-based literature data. Astron. Astrophys. 2013, doi:10.1051/0004-6361/201321956.

50. Pilyugin, L.S.; Vílchez, J.M.; Thuan, T.X. New Improved Calibration Relations for the Determination of Electron Temperatures and Oxygen and Nitrogen Abundances in H II Regions. Astrophys. J. 2010, 720, 1738-1751.

51. Pilyugin, L.S.; Grebel, E.K.; Mattsson, L. "Counterpart" method for abundance determinations in H II regions. Mon. Not. R. Astron. Soc. 2012, 424, 2316-2329.

52. González Delgado, R.M.; Cid Fernandes, R.; García-Benito, R.; Pérez, E.; de Amorim, A.L.; Cortijo-Ferrero, C.; Lacerda, E.A.D.; López Fernández, R.; Sánchez, S. F.; Vale Asari, N.; et al. Insights on the Stellar Mass-Metallicity Relation from the CALIFA Survey Astrophys. J. 2014, 791, L16.

53. Sánchez-Menguiano, L; Sánchez, S.F.; Perez, I.; García-Benito, R.; Husemann, B.; Mast, D.; Mendoza, A.; Ruiz-Lara, T.; Ascasibar, Y.; Bland-Hawthorn, J; et al. Spatially resolved oxygen abundances in CALIFA spiral galaxies Astron. Astrophys. 2015, submitted.

54. Ho, I.T.; Kudritzki, R.P.; Kewley, L.J.; Zahid, H.J.; Dopita, M.A.; Bresolin, F.; Rupke, D.S.N. Metallicity gradients in local field star-forming galaxies: Insights on inflows, outflows, and the coevolution of gas, stars and metals. Mon. Not. R. Astron. Soc. 2015, 448, 2030-2054.

55. Bresolin, F.; Kennicutt, R.C.; Ryan-Weber, E. Gas Metallicities in the Extended Disks of NGC 1512 and NGC 3621. Chemical Signatures of Metal Mixing or Enriched Gas Accretion? 2012, arXiv:astro-ph.CO/1203.0956.

56. pyqz python module extension for MAPPINGS IV simulations. Available online: https://datacommons.anu.edu.au:8443/DataCommons/item/anudc:5037 (accessed on 1 October 2015).

57. Sánchez, S.F.; Pérez, E.; Rosales-Ortega, F.F.; Miralles-Caballero, D.; López-Sánchez, A.R.; Iglesias-Páramo, J.; Marino, R.A.; Sánchez-Menguiano, L.; García-Benito, R.; Mast, D.; et al. Imprints of galaxy evolution on H II regions. Memory of the past uncovered by the CALIFA survey. Astron. Astrophys. 2015, doi:10.1051/0004-6361/201424873.

58. Evans, I.N.; Dopita, M.A. Theoretical models for H II regions. I-Diagnostic diagrams. Astrophys. J. Suppl. 1985, 58, 125-142.

59. Pérez-Montero, E. Deriving model-based $\mathrm{T}_{e}$-consistent chemical abundances in ionised gaseous nebulae. 2014, arXiv:1404.3936.

60. Marino, R.A.; Gil de Paz, A.; Sánchez, S.F.; Sánchez-Blazquez, P.; Cardiel, N.; Castillo-Morales, A.; Pascual, S.; Vílchez, J.; Kehrig, C.; Mollá, M.; et al. Outer-disk reddening and gas-phase metallicities: The CALIFA connection. 2015, arXiv:1509.07878.

61. Ruiz-Lara, T.; Pérez I. ; Florido E.; Sánchez-Blázquez; P.; Méndez-Abreu, J.; Lyubenova, M.; Falcón-Barroso, J.; Sánchez-Menguiano, L.; Sánchez. S.F.; Galbany, L.; et al. No direct coupling between bending of galaxy disc stellar age and light profiles Mon. Not. R. Astron. Soc. 2015, accepted. 
62. Diaz, A.I. Abundance gradients in disc galaxies and chemical evolution models. In Evolutionary Phenomena in Galaxies; Beckman, J.E., Pagel, B.E.J., Eds.; Cambridge University Press: Cambridge, UK, 1989; pp. 377-397.

63. Vila-Costas, M.B.; Edmunds, M.G. The relation between abundance gradients and the physical properties of spiral galaxies. Mon. Not. R. Astron. Soc. 1992, 259, 121-145.

64. Marino, R.A.; Gil de Paz, A.; Castillo-Morales, J.C.; Muñoz-Mateos, S.F.; Sánchez, P.G.; Pérez-González, J.; Gallego, J.; Zamorano, A.; Alonso-Herrero, A.; Boissier, S. Integral Field Spectroscopy and Multi-wavelength Imaging of the nearby Spiral Galaxy NGC 5668: An Unusual Flattening in Metallicity Gradient. Astrophys. J. 2012, doi:10.1088/0004-637X/754/1/61.

65. Tsujimoto, T.; Bland-Hawthorn, J.; Freeman, K.C. Evidence of Early Enrichment of the Galactic Disk by Large-Scale Winds. Publ. Astron. Soc. Jpn. 2010, 62, 447-456.

66. Matteucci, F.; Francois, P. Galactic chemical evolution-Abundance gradients of individual elements. Mon. Not. R. Astron. Soc. 1989, 239, 885-904.

67. Boissier, S.; Prantzos, N. Chemo-spectrophotometric evolution of spiral galaxies-I. The model and the Milky Way. Mon. Not. R. Astron. Soc. 1999, 307, 857-876.

68. Muñoz-Mateos, J.C.; Gil de Paz, A.; Boissier, S.; Zamorano, J.; Jarrett, T.; Gallego, J.; Madore, B.F. Specific Star Formation Rate Profiles in Nearby Spiral Galaxies: Quantifying the Inside-Out Formation of Disks. Astrophys. J. 2007, 658, 1006-1026.

69. Trujillo, I.; Rudnick, G.; Rix, H.W.; Labbé, I.; Franx, M.; Daddi, E.; van Dokkum, P.G.; Förster Schreiber, N.M.; Kuijken, K.; et al. The Luminosity-Size and Mass-Size Relations of Galaxies out to $\mathrm{z} \sim 3$. Astrophys. J. 2004, 604, 521-533.

70. Barden, M.; Rix, H.W.; Somerville, R.S.; Bell, E.F.; Häußler, B.; Peng, C.Y.; Borch, A.; Beckwith, S.V.W.; Caldwell, J.A.R.; Heymans, C.; et al. GEMS: The Surface Brightness and Surface Mass Density Evolution of Disk Galaxies. Astrophys. J. 2005, 635, 959-981.

71. Trujillo, I.; Förster Schreiber, N.M.; Rudnick, G.; Barden, M.; Franx, M.; Rix, H.-W.; Caldwell, J.A.R.; McIntosh, D. H.; Toft, S.; Häussler, B. et al. The Size Evolution of Galaxies since z 3: Combining SDSS, GEMS, and FIRES. Astrophys. J. 2006, 650, 18-41.

72. Pagel, B.E.J.; Patchett, B.E. Metal abundances in nearby stars and the chemical history of the solar neighborhood. Mon. Not. R. Astron. Soc. 1975, 172, 13-40.

73. Gibson, B.K.; Fenner, Y.; Renda, A.; Kawata, D.; Lee, H.C. Galactic Chemical Evolution. Publ. Astron. Soc. Aust. 2003, 20, 401-415.

74. Larson, R.B. Effects of supernovae on the early evolution of galaxies. Mon. Not. R. Astron. Soc. 1974, 169, 229-246.

75. Silk, J. Dissipative Processes in Galaxy Formation. Proc. Natl. Acad. Sci. USA 1993, 90, 4835-4839.

76. Veilleux, S.; Cecil, G.; Bland-Hawthorn, J. Galactic Winds. Annu. Rev. Astron. Astr. 2005, 43, 769-826.

77. Shapley, A.E.; Steidel, C.C.; Pettini, M.; Adelberger, K.L. Rest-Frame Ultraviolet Spectra of z 3 Lyman Break Galaxies. Astrophys. J. 2003, 588, 65-89. 
78. Weiner, B.J.; Coil, A.L.; Prochaska, J.X.; Newman, J.A.; Cooper, M.C.; Bundy, K.; Conselice, C.J.; Dutton, A.A.; Faber, S.M.; Koo, D.C.; et al. Ubiquitous Outflows in DEEP2 Spectra of Star-Forming Galaxies at $\mathrm{z}=1.4$. Astrophys. J. 2009, 692, 187-211.

79. Dekel, A.; Zolotov, A.; Tweed, D.; Cacciato, M.; Ceverino, D.; Primack, J.R. Toy models for galaxy formation versus simulations. Mon. Not. R. Astron. Soc. 2013, 435, 999-1019.

80. Lilly, S.J.; Carollo, C.M.; Pipino, A.; Renzini, A.; Peng, Y. Gas Regulation of Galaxies: The Evolution of the Cosmic Specific Star Formation Rate, the Metallicity-Mass-Star-formation Rate Relation, and the Stellar Content of Halos. Astrophys. J. 2013, 772, 119.

81. Kewley, L.J.; Rupke, D.; Zahid, H.J.; Geller, M.J.; Barton, E.J. Metallicity Gradients and Gas Flows in Galaxy Pairs. Astrophys. J. Lett. 2010, 721, L48-L52.

82. Rich, J.A.; Torrey, P.; Kewley, L.J.; Dopita, M.A.; Rupke, D.S.N. An Integral Field Study of Abundance Gradients in nearby Luminous Infrared Galaxies. Astrophys. J. 2012, doi:10.1088/0004-637X/753/1/5.

(C) 2015 by the authors; licensee MDPI, Basel, Switzerland. This article is an open access article distributed under the terms and conditions of the Creative Commons Attribution license (http://creativecommons.org/licenses/by/4.0/). 